\title{
Changes in microbiota during experimental human Rhinovirus infection
}

\author{
J. J. Hofstra ${ }^{1,5^{*}}$, S. Matamoros ${ }^{1}$, M. A. van de Pol ${ }^{2,3}$, B. de Wever ${ }^{1}$, M. W. Tanck ${ }^{4}$, H. Wendt-Knol ${ }^{1}$, M. Deijs ${ }^{1}$, \\ L. van der Hoek ${ }^{1}$, K. C. Wolthers ${ }^{1}$, R. Molenkamp ${ }^{1}$, C. E. Visser ${ }^{1}$, P. J. Sterk ${ }^{3}$, R. Lutter ${ }^{2,3}$ and M. D. de Jong ${ }^{1}$
}

\begin{abstract}
Background: Human Rhinovirus (HRV) is responsible for the majority of common colds and is frequently accompanied by secondary bacterial infections through poorly understood mechanisms. We investigated the effects of experimental human HRV serotype 16 infection on the upper respiratory tract microbiota.

Methods: Six healthy volunteers were infected with HRV16. We performed 165 ribosomal RNA-targeted pyrosequencing on throat swabs taken prior, during and after infection. We compared overall community diversity, phylogenetic structure of the ecosystem and relative abundances of the different bacteria between time points.

Results: During acute infection strong trends towards increases in the relative abundances of Haemophilus parainfluenzae and Neisseria subflava were observed, as well as a weaker trend towards increases of Staphylococcus aureus. No major differences were observed between day- 1 and day 60 , whereas differences between subjects were very high.

Conclusions: HRV16 infection is associated with the increase of three genera known to be associated with secondary infections following HRV infections. The observed changes of upper respiratory tract microbiota could help explain why HRV infection predisposes to bacterial otitis media, sinusitis and pneumonia.
\end{abstract}

\section{Background}

Human Rhinovirus is a globally endemic virus, belonging to the family of Picornaviridae and the most common cause of the common cold. Although usually causing mild to moderate disease, HRV triggers exacerbations of chronic obstructive pulmonary disease, asthma and cystic fibrosis [1, 2], and may cause severe lower respiratory tract disease particularly in risk groups such as infants, the elderly and immunocompromised patients [3]. In addition, infection with HRV is a predisposing factor for secondary bacterial infections (otitis media, sinusitis, pneumonia) [4-7]. Insights into the mechanisms through

\footnotetext{
* Correspondence: j.j.hofstra@amc.uva.nl

This work has been presented at-Cell Symposia: Microbiome and Host Health, Lisbon, Portugal, 2013-The Macrae Foundation's XV International Symposium on Respiratory Viral Infections - Rotterdam, The Netherlands 2013

'Department of Medical Microbiology, Academic Medical Centre, University of Amsterdam, Meibergdreef 9, 1105 AZ Amsterdam, The Netherlands ${ }^{5}$ Department of Anaesthesiology, Academic Medical Centre, University of Amsterdam, Amsterdam, The Netherlands

Full list of author information is available at the end of the article
}

which HRV enhances bacterial supra-infection remain limited.

HRV associated bacterial superinfections are assumed to be caused in part by anatomical and mechanical causes, e.g. due to Eustachian tube dysfunction [8], ostiomeatal obstruction and reduced mucocilliary clearance $[9,10]$. In addition, HRV infection may increase epithelial permeability [11], promote bacterial adherence and entry into the epithelium $[12,13]$ or reduce host responses to bacterial pathogens[14]. Common pathogens associated with HRV-associated bacterial infections include S. pneumoniae, H. influenzae and M. catarrhalis.

In recent years, culture-independent DNA-based techniques demonstrated that highly complex microbial communities are present in the upper airways. These communities are an integral part of our commensal airway microbiota and likely contribute to infection and disease. HRV infection and the subsequent host response [11-14] change the upper airway local environment and will likely alter the microbiota composition. Insights into HRV induced changes in microbiota composition could contribute to our understanding of the pathophysiological 
mechanisms of bacterial super-infection and could help to predict secondary bacterial infections thereby ultimately contributing to preventive strategies and tools. Most human studies to date have been retrospective, showing epidemiological correlations between HRV and the presence of potential respiratory pathogens [15-17]. Such correlation, however, do not necessarily implicate causality and interpretation of these data warrants caution. Most studies of the lung microbiota have been cross-sectional, observational and descriptive in nature [18].

Here we describe the effects of HRV infection on the upper respiratory tract microbiota in otherwise healthy human volunteers experimentally infected with HRV serotype 16 (HRV16), enabling controlled observations before, during and after resolution of infection. We hypothesized that HRV16 infection alters throat bacterial microbiota compositions in healthy volunteers in terms of the bacterial diversity (Shannon and Simpson diversity indexes) as well as bacterial richness (Chao1 measure). We also analysed changes over time in relative abundances of individual bacterial genera/species.

\section{Methods}

\section{Study subjects}

Healthy volunteers (6 in total) aged 18-60 were eligible for participation provided they had not had a common cold during the six weeks prior to enrolment.

Presence of common cold was defined as a cumulative validated cold symptom score [19] of $\geq 14$ over a 6 day period and the subjective impression of a cold, rhinorrhoea on at least three days. Other exclusion criteria were: receipt of BCG-vaccination, chest $\mathrm{x}$-ray abnormalities, a history of lung disease, signs of airway obstruction (Forced Expiratory volume in $1 \mathrm{~s}$ (FEV1) $>80 \%$ of predicted value), seasonal or perennial rhinitis or sinusitis, smoking (unless stopped smoking 12 months prior to the study, max. 5 pack years), any clinically significant abnormality in medical history and clinical examination, participation in any clinical investigational drug treatment protocol within 30 days of enrolment, pregnancy or lactation, any medication usage. The study protocol was approved by the Academic Medical Center Amsterdam ethical review committee. Written informed consent was obtained from all volunteers.

\section{Study design}

In this prospective single center study all volunteers were experimentally infected with HRV16 (Fig. 1). Upon enrolment the volunteers were screened to ensure the absence of circulating antibodies against tuberculosis, HRV16, Human T-lymphotropic virus Type 1 (HTLV-1) and 2 (HTLV-2), Human Immunodeficiency virus (HIV), and Hepatitis A, B and C virus (HAV, HBV, HCV). Acute infection with Influenza $A$ and $B$, enterovirus sp., adenovirus sp., rhinovirus sp., human metapneumovirus, human respiratory syncytial virus sp., parainfluenza viruses 1-4, human parechovirus, human bocavirus, coronavirus sp., Chlamydia pneumoniae, Mycoplasma pneumoniae, legionella $s p$. was excluded through validated PCR assays on nasal lavage and throat swabs. These throat swabs were collected to serve as baseline microbiota measures. One day later volunteers were experimentally infected with HRV16 (details below), which has been shown to cause mild common-cold symptoms. Nasal lavages and throat swabs were performed daily for 7 days. On day 60 a convalescent throat swab was taken. All volunteers were requested to report common cold and asthma symptoms daily until day 7 after rhinovirus challenge using a standardized cold symptom score [20]. Diaries were collected at the final visit.

\section{HRV16 challenge}

Experimental HRV16 infection was induced as described previously [21]. In short, $100 \mu \mathrm{l}$ of an HRV16 aliquot (stored at $-80^{\circ} \mathrm{C}$ ) was kept on ice, thawed and diluted in sterile $0.9 \% \mathrm{NaCl}$ just before exposure. $750 \mu \mathrm{l}$ of this dilution was transferred to a sterile micronic tube positioned in a glass support of the DeVilbiss 286 atomizer (DeVilbiss Healthcare, Somerset, PA, USA). This dilution was administered to the healthy volunteer by spray in one nostril while closing off the other nostril. The total administered HRV16 dose was $10 \mathrm{TCID}_{50}$. Infection was confirmed by positive PCRs, positive viral cultures and development of symptoms.

\section{Nasal lavage}

One nostril was closed off using an inflatable catheter as described previously [20].

Slowly, $10 \mathrm{ml}$ of sterile Hanks balanced salt solution (without phenol) at body temperature was instilled via the catheter and left for $5 \mathrm{~min}$. Lavage fluid was collected in tube for viral detection. The procedure was repeated in the other nostril. All used materials were sterile or disinfected using $70 \%$ ethanol prior to use.

\section{Throat swabs}

A cotton-tipped swab (Copan Diagnostics, Corona, Canada) was used to obtain material from the space between the palatine arches. The cotton swab was transferred to universal transport medium (UTM, Copan Diagnostics) in a tightly closed tube. After vortexing, the swab was discarded and the medium was stored at $-80{ }^{\circ} \mathrm{C}$.

Infection was confirmed by HRV-positive quantitative PCR in nasal and/or throat samples as described elsewhere [19]. In addition, presence of replication-competent HRV was confirmed by virus culture (human embryonic lung cells). 


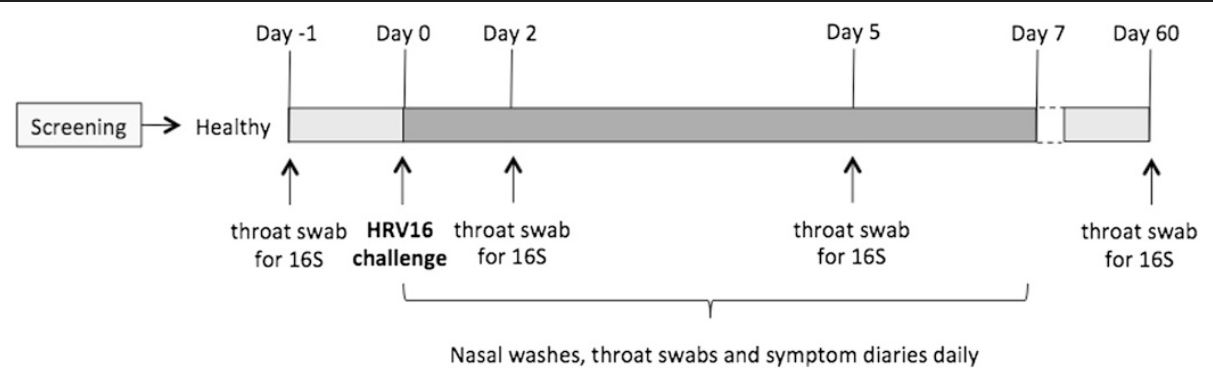

Fig. 1 Study design. Screening was performed on the day before experimental rhinovirus infections. Nasal washes and throat swabs for Human Rhinovirus (HRV) PCR and viral culturing were repeated daily from day 0 until day 7 . A throat swab for 165 rRNA gene sequencing was collected prior to HRV16 challenge (day -1) and subsequently on days 2, 5, and day 60

\section{5 rRNA gene analysis}

After treatment with an in-house enzymatic cocktail (achromopeptidase, mutanolysine, lysostaphine (1000:100:3) and lysosyme $(1 \mathrm{mg} / \mathrm{ml})$ (Sigma-Aldrich, St. Louis, MO, USA) in TE-buffer) as well as an in-house lysis buffer (Sodium-docecyl-Sulphate (1\%), Tween-20 (0.5\%) and Sarkosyl (0.5 \%) in TE-buffer), DNA was extracted using the MagnaPure LC (Roche Diagnostics, Indianapolis, IN, USA) following the manufacturer's instructions. Bacterial rRNA genes were quantified by rt-PCR using primers F-16S-27 (5'-AGAGTTTGATCCTGGCTCA G-3') and R-16S-355 (5' - GCTGCCTCCCGTAGGAGT-3') which bind to an area of the $16 \mathrm{~S}$ rRNA gene conserved in all bacteria [26, 27], and probe P-16S (6FAM-CTGGCGGCRKGCYTAACACATGCAAGTCGABHQ1). Subsequently, 16S rRNA genes were amplified using the primers detailed in the supplementary material followed by a second round of PCR using primers with adapters and barcodes (see Additional file 1 for full description).

The amplified fragments with adapters and tags were quantified using the Quant-iT dsDNA Assay Kit on a Qubit fluorometer (Invitrogen/Life Technologies, Carlsbad, CA, USA). Emulsion PCR was performed according to the protocol (emPCR Method Manual - Lib-A SV jan2010) supplied with the GS FLX Titanium XLR 70 Sequencing kit (Roche Diagnostics, Indianapolis, IN, USA). Two- region 454 sequencing run was performed on a GS FLX Titanium Pico TiterPlate $(70 \times 75)$ using a GS FLX Titanium Sequencing Kit XLR70 according to the manufacturer's instructions (Roche Diagnostics, Indianapolis, IN, USA).

\section{Data processing and analysis}

Data analysis was performed using the software "Quantitative Insights into Microbial Ecology" (QIIME 1.8.0) [22]. After removal of low quality reads (quality score $<25$ ) and chimera (ChimeraSlayer) our data was denoised using the QIIME denoiser program [23]. Then we clustered the sequences into Operational Taxonomical Units (OTU's) based on $97 \%$ sequence similarity (Uclust) [24]. The resulting OTU table was then condensed by removing all OTUs representing less than $0.005 \%$ of the total number of sequences [25]. Uclust classifier was used to assign taxonomy. FastTree was used to construct a phylogenetic tree for downstream analyses.

\section{Community structure}

Bacterial taxonomic richness and diversity were determined using rarefaction plots of the normalized number of sequences per time point. Rarefaction curves were generated for $3 \%$ genetic difference level (e.g. at the genus level). The Shannon diversity measure is a quantitative measure that reflects the number of different species simultaneously taking into account how evenly the bacteria are distributed among those species [26]. Another method to examine the microbiota community structure is to use the Chaol measure which estimates the minimum richness in each sample [27]. Chaol, by taking into account the number of species found only once (singletons) or twice (doubletons) in a sample, provides an estimation of the number of undetected species in the microbiota, which is important as the human associated microbiota contains many rare species.

UniFrac is a method to calculate a distance measure between samples using the phylogenetic trees. The UniFrac measure expresses the fraction of shared branches in the phylogenetic trees between samples [28].

Ecological intra- and inter-patient microbiota similarity was estimated using adonis, ANOSIM and MRPP methods included in QIIME 1.8.0. Briefly, these methods compare the ecological distance between samples (Unifrac distance) to test the null hypothesis that intragroup similarity is not significantly different than intergroup similarity [29].

\section{Statistical analysis}

With a sample size $(n=6)$ we expected to be able to detect an effect of $25 \%$ on the bacterial richness measure Chao1 with two-sided significance of $\mathrm{p}=0.05$ and a power of $90 \%$, assuming a standard deviation of $20 \%$. We normalized the data by calculating the OTU proportion-the 
proportion of total sequences per microbial community, i.e., per sample. To compare differences in microbiota members between time points we used a linear mixed model. Microbial community comparisons were performed using parametric statistics in $\mathrm{R}$, with the $\mathrm{P}$ values corrected by multiple hypothesis testing using the false discovery rate (FDR) [30]. To assess differences in overall community structure the data of all volunteers were pooled per time point and diversity measures (alpha diversity measures Shannon index, Simpson index and Chao1) [28]. Considering that the alpha diversity indices (i.e. Shannon diversity and Chao1) are sensitive to the original number of sequences generated from a given sample [31] we calculated the Shannon and Simpson diversity and Chaol for normalized numbers of sequences for each separate sample. A number of randomly picked reads, corresponding to the lowest number of sequences in a sample group was picked 100 times from each sequence set.

\section{Results}

\section{Clinical data}

In total 6 volunteers ( 3 males, 3 females) were included in the study (age range 18-28 years). HRV16 infection was established as demonstrated by positive HRV PCRs, positive viral cultures (data not shown) and development of symptoms in all. Viral RNA loads of nasal washes and throat swabs and the cold symptom scores are shown in Fig. 2.

\section{$16 \mathrm{~S}$ analysis results}

After removing sequences representing less than $0.005 \%$ of the total abundance, the median number of sequences per sample was 5407 (min.: 2760; max.: 8589; sd: 1283.3). When sequences were clustered at $97 \%$ sequence identity 384 operational taxonomical units (OTUs) were identified. Taxonomic analysis identified 11 bacterial phyla, containing 80 genera, 53 of which were successfully identified with $>80 \%$ confidence. The most commonly amplified bacterial phyla (Proteobacteria, Bacteroidetes, Actinobacteria, and Firmicutes) did not vary significantly over time
(Fig. 3). Streptococcus, Prevotella, Veillonella, Rothia, and Haemophilus were the 5 most common bacterial genera amplified, together accounting for $63.8 \%$ of the total number of sequences. These genera contained 101 species level OTUs, 20 of which were successfully identified ( $>90 \%$ sequence similarity).

\section{Community structure}

Alpha diversity measures (Chao1, Shannon and Simpson indexes) were similar in specimens obtained at baseline or convalescence (days -1 and 60 ) and during infection (days 2 and 5).

A principle coordinates analysis based on the weighted UniFrac measure (fraction of shared branches in a phylogenetic tree) revealed that samples seem to cluster by individual volunteer although there was substantial overlap between individuals (Fig. 4). All 3 measures of ecological clustering (Adonis, ANOSIM and MRPP) showed that intra-individual UniFrac distance between samples was lower than inter-individual sample distance. Analysis on the data pooled by time point based on the UniFrac measure revealed no significant differences between time points (weighted UniFrac significance $\mathrm{p}>$ 0.05 , data not shown) nor were time points 2 and 5 (during infection) significantly different from time points -1 (pre-infection) and 60 (convalescence). Using the UniFrac distance measure to assess changes in microbiota composition of each individual from its own baseline also revealed no significant differences between any time points (Fig. 4).

\section{Individual genera/species}

Among the 5 most abundantly identified genera, three (Streptococcus, Prevotella and Rothia) showed very limited variation during HRV16 infection (less than $10 \%$ of the baseline abundance), whereas Veillonella decreased by approximately $25 \%$ and Haemophilus increased by $75 \%$.

Paired comparisons using a linear mixed model were used to assess changes over time within individuals, days -1 and 60 being grouped as baseline non-infection measures,
Cold Symptom Score

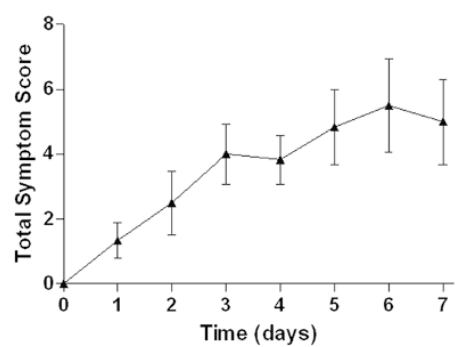

Viral Titers

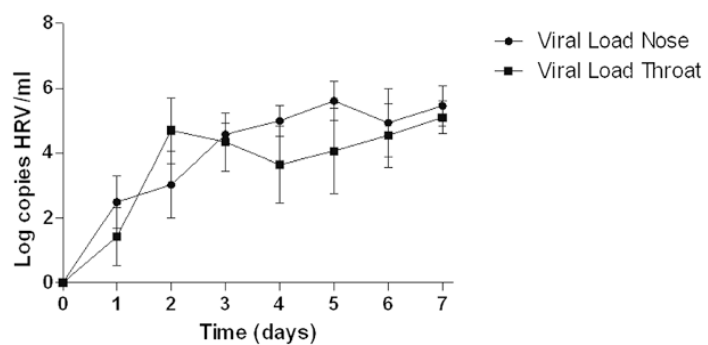

Fig. 2 Human Rhinovirus Infection. Representation of the total Cold Symptom Scores as assessed by a daily questionnaire [1] as well as viral loads measured through RT-PCR in nasal lavage fluid and throat swabs of healthy volunteers after nasal inoculation of Human Rhinovirus serotype $16($ HRV-16) 
Actinobacteria

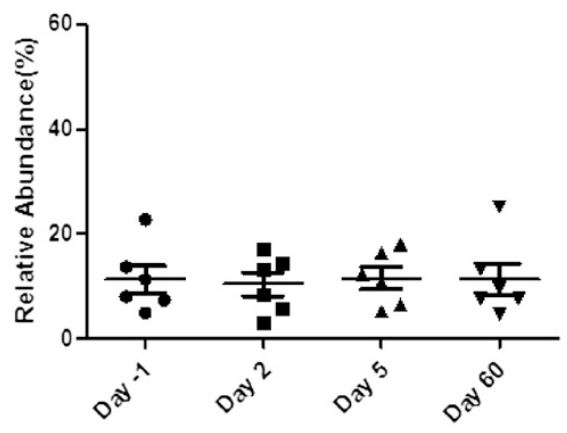

Firmicutes

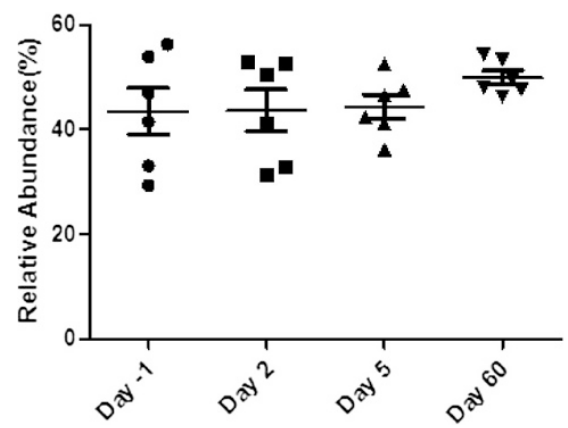

Bacteriodetes

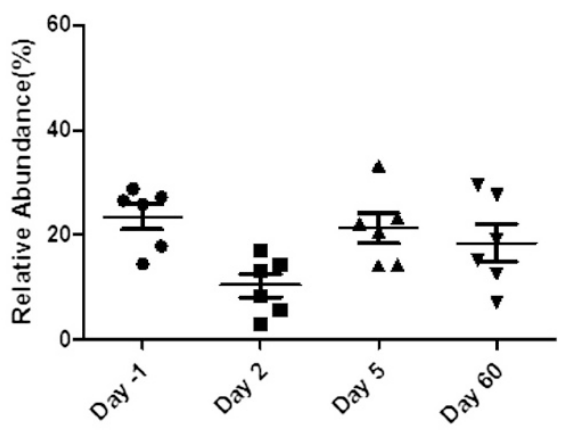

Proteobacteria

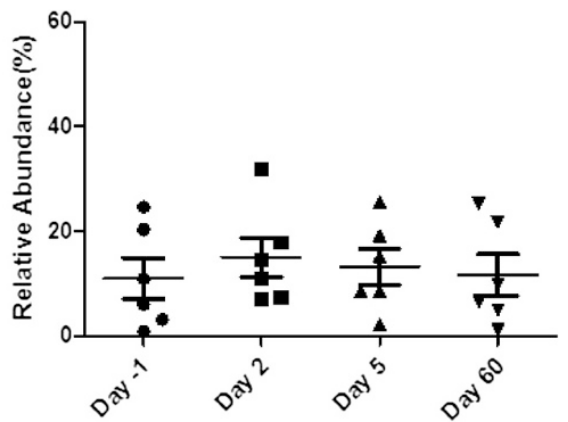

Fig. 3 Bacterial Phyla. The distribution (and means \pm SEM) of the top four phyla grouped by each time point: (day -1 (circles), day 2 (squares), day 5 (triangles pointing up), day 60 (triangles pointing down). No significant changes from baseline were observed in any of the phyla following HRV-16 infection ( $p>0.05$ across time points for all phyla using repeated measures ANOVA)

and days 2 and 5 being grouped as infection measures. No significant changes were observed between day -1 and day 60 specimens at genus or species level. However, at the genus level, a significant increase $(\mathrm{p}<0.05)$ in relative abundance of Haemophilus and Neisseria was observed during infection. Analysis of these genera to species level show significantly increased Haemophilus parainfluenzae ( $\mathrm{p}$-value $=0.0098)$ and Neisseria subflava ( $\mathrm{p}$-value $=$ 0.012 ). However, false discovery rate (FDR) correction for multiple comparisons showed an above threshold risk of type-I error for all these taxa (FDR =0.12). Additionally, the largest fold-change observed was a $1200 \%$ (13-fold) increase in the abundance of the genus Staphylococcus ( $\mathrm{p}=0.0749$ FDR 0.518), which only represents $0.2 \%$ of the total sequences identified (Fig. 5). Analysis to species level shows a weak trend towards increases in Staphylococcus aureus abundance $(\mathrm{p}$-value $=0.0856 \mathrm{FDR}=0.245)$.

The increases in $H$. parainfluenzae during infection were observed in all volunteers, while the Neisseria species increased in 5 of 6 volunteers. Staphylococcus aureus was only present in four volunteers and increased in three, hence the observed correlation between HRV16 and Staphylococcus aureus was the weakest ( $\mathrm{p}$-value $=0.0856 \mathrm{FDR}=0.245)$. Observed changes in the microbiota composition did not correlate with viral loads or symptom scores (data not shown).

\section{Discussion}

In this study we demonstrate for the first time that infection with HRV16 in otherwise healthy volunteers alters upper respiratory tract microbiota composition. Although the overall changes are moderate, increases in relative abundances of the bacterial genera Haemophilus, Staphylococcus and Neisseria were observed. At a species level, increases were observed of Haemophilus parainfluenzae, Staphylococcus aureus and Neisseria subflava species. These increases in genera and species during HRV16 infection were observed in nearly all volunteers who carried these at baseline and relative abundances returned to baseline levels after the infection was cleared. Due to the limited number of study subjects $(n=6)$ and the stringent correction for multiple observations, these changes did not reach statistical significance. Nevertheless, it is striking that from over one hundred genera detected, substantial increases are recorded for 2 genera generally associated with secondary bacterial infections after HRV16 infection (Heamophilus and Staphylococcus).

These findings suggest that changes in the composition of resident microbiota per se may play a role in the 


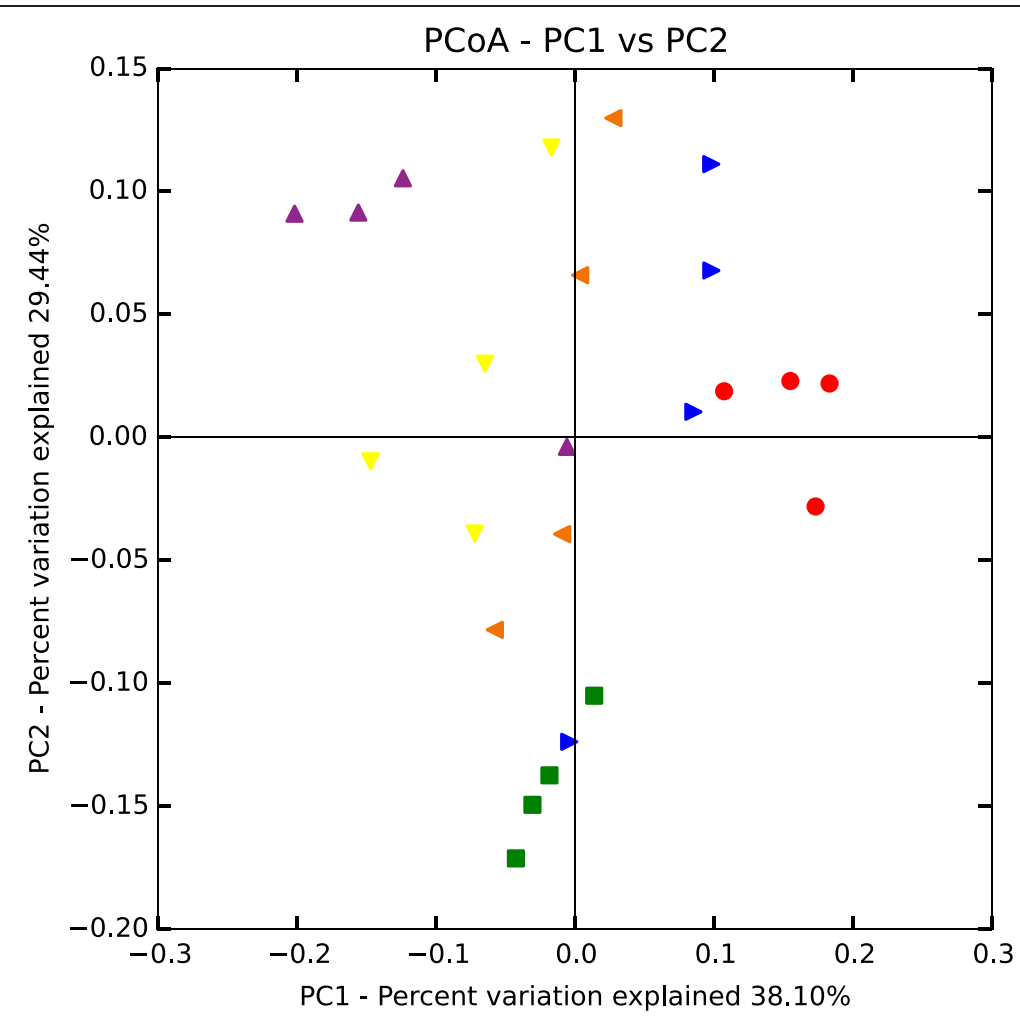

Fig. 4 Principle component analysis (Unifrac measure). Samples coloured by individual: volunteer 1 (red triangles), volunteer 2 (blue triangles), volunteer 3 (brown triangles), volunteer 4(green circles), volunteer 5 (purple squares), volunteer 6 (yellow triangles). Generally samples seem to cluster by individual although there is substantial overlap between individuals. There were no significant changes over time with (weighted Unifrac significance). There were no significant differences between infected vs non-infected samples (weighted Unifrac significance)

development of bacterial super-infections associated with HRV infection. Our data extend previous findings by Molyneaux et al., who described similar effects of experimental rhinovirus infection on the sputum microbiota in COPD patients [32] where in particular increases in Haemophilus sp. and Neisseria sp. were observed. However, in contrast with our findings, the microbiota of HRV infected healthy volunteers (i.e. without COPD) from the study by Molyneaux et al. did not seem to be affected [32] which may be explained by differences in study design, e.g. the use of sputum specimens vs. throat swabs. Interestingly, in asymptomatic Aboriginal children the presence of HRV was significantly associated with the presence of $H$. influenzae and $M$. catarrhalis in nasopharyngeal aspirates [15]. In a Dutch cohort of otitis-prone children, HRV positivity was significantly associated with the presence of Streptococcus pneumoniae and M. catarrhalis in the nasopharynx, but not with that of $H$. influenzae [17]. A later study in healthy children showed that $H$. influenzae was positively associated with the presence of HRV [16].

In vitro studies have demonstrated that infection with HRV induces changes in our microbiota's habitat through augmentation of the adhesion of bacterial pathogens to the airway epithelium [11-13, 33, 34].
Given the complexity of the upper airway ecosystem it seems likely that direct viro-bacterial as well as bacterial-bacterial interactions may also play a role. Although a number of these direct interactions have been identified, their role during HRV infection remains to be elucidated [35]. The present new data on cultureindependent classification of bacteria present in the throat during upper respiratory tract infection contributes to the understanding of upper respiratory tract microbial communities during HRV16 infection, and possible polymicrobial interactions.

Despite the statistical limitations mentioned above the observed trends occur in nearly all individual and involve 'usual suspects' for secondary bacterial infections. As the available volunteers for our study were relatively young extrapolation of these findings to other age groups should be done with caution.

Strengths of our study are the stringent exclusion of the presence of any other respiratory pathogens and our culture independent next generation sequencing approach, allowing sufficient depth to identify even the rarer species in our samples. However, deeper sequencing might have revealed minor other differences in the community composition [31]. Furthermore, our study 


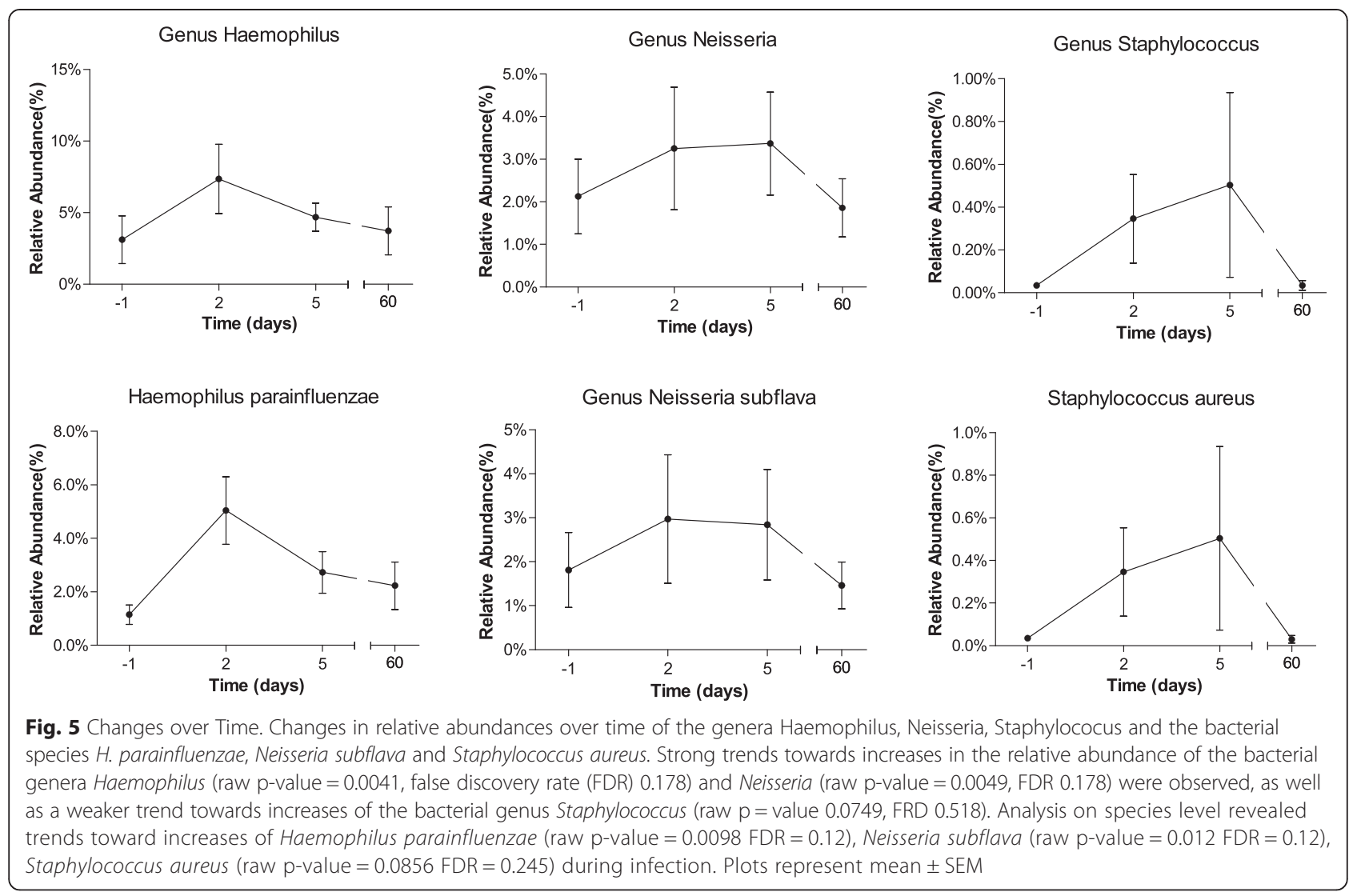

was underpowered to investigate differences in individual bacterial species in the upper respiratory tract microbiota. Some limitations are inherent to 454 sequencing of $16 \mathrm{~S}$ genes in general. They include possible bias introduced by the extraction, amplification and sequencing methodologies. Finally the number of genomic $16 \mathrm{~S}$ copies varies considerably from bacteria to bacteria. Currently there is insufficient knowledge about exact $16 \mathrm{~S}$ copy numbers to correct for this [36].

Although we were able to observe changes within each volunteer before, during and after HRV16 infection, we did not include a group of uninfected volunteers. However since our volunteers functioned as their own controls and the human microbiota during health has been shown to be relatively stable over time [37] it is likely that the observed microbiota changes were induced by HRV16 infection although we cannot exclude the possibility the daily collection of throat swabs itself may have affected microbiota composition. Our approach reduces the background noise inherent to human microbiota studies, which is mainly due to the large differences between individuals. This way we expect a more precise detection of relevant changes in the microbiota.

The fact that we used sequential samples allowed us to use the linear mixed model on non-independent (paired) samples, which focusses on the response of the variables of interest (bacterial taxa) during the course of the experiment and minimizes background noise of the high variability between individuals.

Our findings have clinical relevance and may contribute to further understanding of the early stages in the development of bacterial complications, such as acute otitis media, sinusitis and pneumonia, following HRV and other respiratory viral infections [4]. Larger controlled serially sampled studies in experimental and naturally occurring HRV infections are warranted to confirm and extend our current observations. It would be of clinical interest to investigate whether clinical secondary bacterial infections after HRV infection are actually preceded by similar changes in microbiota composition.

\section{Conclusions}

In conclusion our study showed HRV16 infection is associated with strong trends towards increases in the relative abundances of Haemophilus and Neisseria and a weaker trend towards increases in the relative abundance of Staphylococcus. Even if the observed changes of upper respiratory tract microbiota are minor overall, they may be of great clinical significance and could help explain why HRV infection predisposes to bacterial otitis media, sinusitis and pneumonia. 


\section{Additional file}

Additional file 1: Bacterial 16S rRNA Gene Sequencing. (DOCX $32 \mathrm{~kb}$ )

\section{Abbreviations}

FDR: False Discovery Rate; FEV1: Forced Expiratory Volume in $1 \mathrm{~s}$; HAV: Hepatitis A Virus; HBV: Hepatitis B Virus; HCV: Hepatitis C Virus; HIV: Human Immunodeficiency Virus; HTLV-1: Human T-Iymphotropic virus Type 1; HTLV-2: Human T-lymphotropic virus Type 2; HRV: Human Rhinovirus; HRV16: Human Rhinovirus serotype 16; OTU: Operational Taxonomical Unit; QIIME: Quantitative Insights into Microbial Ecology; UTM: Universal Transport Medium.

\section{Competing interests}

The authors declare that they have no competing interests.

\section{Authors' contributions}

$J H, S M, B d e W$ and HWK analysed the data. JH and SM performed statistical analysis and drafted the manuscript. MvdP, PS, RL, and MdeJ designed and performed the healthy volunteer study. KW, RM, BdeW, HWK and CV did viral and bacterial analyses on the clinical volunteer samples. BdeW and HWK designed the $16 \mathrm{~S}$ library preparation and participated in the 454 sequencing efforts and participated in the analysis of the sequence data. MT assisted in the statistical analysis of the sequence data. MD and LvdH participated in library preparation and 454 sequencing efforts. All authors participated in drafting the manuscript and read and approved the final manuscript.

\section{Acknowledgements}

This work received funding from the Innovative Medicines Initiative (IMI) under the project U-BIOPRED (Grant Agreement No 115010) and from the European Union Seventh Framework Programme (FP7) under the project PREPARE (grant agreement No 602525).

\section{Author details}

${ }^{1}$ Department of Medical Microbiology, Academic Medical Centre, University of Amsterdam, Meibergdreef 9, 1105 AZ Amsterdam, The Netherlands. ${ }^{2}$ Department of Experimental Immunology, Academic Medical Centre, University of Amsterdam, Amsterdam, The Netherlands. ${ }^{3}$ Department of Respiratory Medicine, Academic Medical Centre, University of Amsterdam, Amsterdam, The Netherlands. ${ }^{4}$ Department of Clinical Epidemiology, Biostatistics and Bioinformatics, Academic Medical Centre, University of Amsterdam, Amsterdam, The Netherlands. ${ }^{5}$ Department of Anaesthesiology, Academic Medical Centre, University of Amsterdam, Amsterdam, The Netherlands.

\section{Received: 19 February 2015 Accepted: 4 August 2015}

\section{Published online: 14 August 2015}

\section{References}

1. Smyth AR, Smyth RL, Tong CY, Hart CA, Heaf DP. Effect of respiratory virus infections including rhinovirus on clinical status in cystic fibrosis. Arch Dis Child. 1995;73:117-20.

2. Wedzicha JA. Role of viruses in exacerbations of chronic obstructive pulmonary disease. Proc Am Thorac Soc. 2004;1:115-20.

3. Jacobs SE, Lamson DM, Kirsten S, Walsh TJ. Human rhinoviruses. Clin Microbiol Rev. 2013;26:135-62.

4. Henderson FW, Collier AM, Sanyal MA, Watkins JM, Fairclough DL, Clyde WA, et al. A longitudinal study of respiratory viruses and bacteria in the etiology of acute otitis media with effusion. N Engl J Med. 1982;306:1377-83.

5. Lehtinen $P$, Jartti $T$, Virkki $R$, Vuorinen $T$, Leinonen $M$, Peltola $V$, et al. Bacterial coinfections in children with viral wheezing. Eur J Clin Microbiol Infect Dis. 2006;25:463-9

6. Louie JK, Roy-Burman A, Guardia-Labar L, Boston EJ, Kiang D, Padilla T, et al. Rhinovirus associated with severe lower respiratory tract infections in children. Pediatr Infect Dis J. 2009:28:337-9.

7. Massa HM, Cripps AW, Lehmann D. Otitis media: viruses, bacteria, biofilms and vaccines. Med J Aust. 2009:191(9 Suppl):S44-9.

8. McBride TP, Doyle WJ, Hayden FG, Gwaltney JM. Alterations of the eustachian tube, middle ear, and nose in rhinovirus infection. Arch Otolaryngol Head Neck Surg. 1989;115:1054-9.
9. Doyle WJ, Skoner DP, Fireman P, Seroky JT, Green I, Ruben F, et al. Rhinovirus 39 infection in allergic and nonallergic subjects. J Allergy Clin Immunol. 1992;89:968-78.

10. Gwaltney JM. Acute Community-Acquired Sinusitis. Clin Infect Dis. 1996;23:1209-25

11. Sajjan U, Wang Q, Zhao Y, Gruenert DC, Hershenson MB. Rhinovirus disrupts the barrier function of polarized airway epithelial cells. Am J Respir Crit Care Med. 2008;178:1271-81.

12. Ishizuka S, Yamaya M, Suzuki T, Takahashi H, Ida S, Sasaki T, et al. Effects of rhinovirus infection on the adherence of Streptococcus pneumoniae to cultured human airway epithelial cells. J Infect Dis. 2003;188:1928-39.

13. Wang JH, Kwon HJ, Jang YJ. Rhinovirus enhances various bacterial adhesions to nasal epithelial cells simultaneously. Laryngoscope. 2009:119:1406-11.

14. Mallia P, Footitt J, Sotero R, Jepson A, Contoli M, Trujillo-Torralbo M-B, et al. Rhinovirus infection induces degradation of antimicrobial peptides and secondary bacterial infection in chronic obstructive pulmonary disease. Am J Respir Crit Care Med. 2012;186:1117-24.

15. Moore HC, Jacoby P, Taylor A, Harnett G, Bowman J, Riley TV, et al. The interaction between respiratory viruses and pathogenic bacteria in the upper respiratory tract of asymptomatic Aboriginal and non-Aboriginal children. Pediatr Infect Dis J. 2010;29:540-5.

16. van den Bergh MR, Biesbroek G, Rossen JWA, de Steenhuijsen Piters WAA, Bosch AATM, van Gils EJM, et al. Associations between pathogens in the upper respiratory tract of young children: interplay between viruses and bacteria. PLoS One. 2012;7:e47711.

17. Pitkäranta A, Roivainen M, Blomgren K, Peltola J, Kaijalainen T, Räty R, et al. Presence of viral and bacterial pathogens in the nasopharynx of otitis-prone children. A prospective study. Int J Pediatr Otorhinolaryngol. 2006;70:647-54.

18. Dickson RP, Erb-Downward JR, Huffnagle GB. The role of the bacterial microbiome in lung disease. Expert Rev Respir Med. 2013;7:245-57.

19. Jansen RR, Wieringa J, Koekkoek SM, Visser CE, Pajkrt D, Molenkamp R, et al. Frequent detection of respiratory viruses without symptoms: toward defining clinically relevant cutoff values. J Clin Microbiol. 2011:49:2631-6.

20. Grünberg K, Timmers MC, Smits HH, de Klerk EP, Dick EC, Spaan WJ, et al. Effect of experimental rhinovirus 16 colds on airway hyperresponsiveness to histamine and interleukin-8 in nasal lavage in asthmatic subjects in vivo. Clin Exp Allergy. 1997;27:36-45.

21. Van der Sluijs KF, van de Pol MA, Kulik W, Dijkhuis A, Smids BS, van Eijk HW, et al. Systemic tryptophan and kynurenine catabolite levels relate to severity of rhinovirus-induced asthma exacerbation: a prospective study with a parallel-group design. Thorax. 2013;68(Wolthers KC, Johnston SL, van der Zee JS):1122-30

22. Caporaso JG, Kuczynski J, Stombaugh J, Bittinger K, Bushman FD, Costello EK, et al. QIIME allows analysis of high-throughput community sequencing data. Nat Methods. 2010;7:335-6.

23. Reeder J, Knight R. Rapid denoising of pyrosequencing amplicon data: exploiting the rank-abundance distribution. Nat Methods. 2010;7:668-9.

24. Edgar RC. Search and clustering orders of magnitude faster than BLAST. Bioinformatics. 2010;26:2460-1.

25. Bokulich NA, Subramanian S, Faith JJ, Gevers D, Gordon Jl, Knight R, et al. Quality-filtering vastly improves diversity estimates from Illumina amplicon sequencing. Nat Methods. 2013;10:57-9.

26. Shannon CE. The mathematical theory of communication. 1963. MD Comput. 1963:14:306-17.

27. Chao A, Shen T. Nonparametric estimation of Shannon's index of diversity when there are unseen species in sample. In Environ Ecol Stat Volume. 2003;10:429-43.

28. Lozupone C, Hamady M, Knight R. UniFrac-an online tool for comparing microbial community diversity in a phylogenetic context. BMC Bioinformatics. 2006;7:371.

29. Bruce McCune, James B. Grace DLU. Analysis of Ecological Communities. illustrate. MjM Software Design; 2002:300

30. White JR, Nagarajan N, Pop M. Statistical methods for detecting differentially abundant features in clinical metagenomic samples. PLoS Comput Biol. 2009;5:e1000352

31. Lemos LN, Fulthorpe RR, Triplett EW, Roesch LFW. Rethinking microbial diversity analysis in the high throughput sequencing era. J Microbiol Methods. 2011;86:42-51.

32. Molyneaux PL, Mallia P, Cox MJ, Footitt J, Willis-Owen SAG, Homola D, et al. Outgrowth of the Bacterial Airway Microbiome following Rhinovirus 
Exacerbation of Chronic Obstructive Pulmonary Disease. Am J Respir Crit Care Med. 2013;188:1-46.

33. Avadhanula V, Rodriguez CA, Devincenzo JP, Wang Y, Webby RJ, Ulett GC. Respiratory viruses augment the adhesion of bacterial pathogens to respiratory epithelium in a viral species- and cell type-dependent manner. J Virol. 2006;80:1629-36.

34. Wang JH, Kwon H-J, Lee B-J, Jang YJ. Staphylococcal enterotoxins A and B enhance rhinovirus replication in A549 cells. Am J Rhinol. 2013;21:670-4.

35. Bosch AATM, Biesbroek G, Trzcinski K, Sanders EA, Bogaert D. Viral and bacterial interactions in the upper respiratory tract. PLoS Pathog. 2013;9:e1003057.

36. Kembel SW, Wu M, Eisen JA, Green JL. Incorporating 165 gene copy number information improves estimates of microbial diversity and abundance. PLoS Comput Biol. 2012;8:e1002743.

37. Li K, Bihan M, Methé BA. Analyses of the stability and core taxonomic memberships of the human microbiome. PLoS One. 2013;8:e63139.

\section{Submit your next manuscript to BioMed Central and take full advantage of:}

- Convenient online submission

- Thorough peer review

- No space constraints or color figure charges

- Immediate publication on acceptance

- Inclusion in PubMed, CAS, Scopus and Google Scholar

- Research which is freely available for redistribution 